\title{
Floral Characters of Gladiolus as Influenced by Gamma Irradiation
}

\author{
Kiran Kumari ${ }^{1 *}$, Santosh Kumar ${ }^{2}$ and Pragnyashree Mishra ${ }^{3}$ \\ ${ }^{1}$ Department of Floriculture \& Landscape Architecture, College of Horticulture, S.D. \\ Agricultural University, Jagudan, Distt. Mehsana, Gujarat-382 710, India \\ ${ }^{2}$ Department of Horticulture (Floriculture and Landscaping), G.B. Pant University of \\ Agriculture and Technology, Pantnagar, Uttarakhand-263 145, India \\ ${ }^{3}$ College of Horticulture, Odisha University of Agriculture \& Technology, \\ Chiplima, Odisha, India \\ *Corresponding author
}

\begin{tabular}{|c|c|}
\hline & A B S \\
\hline & \multirow{5}{*}{$\begin{array}{l}\text { In the present investigation corms of eight gladiolus varieties (Yellow Golden, Nathan } \\
\text { Red, White Friendship, Jester Gold, American Beauty, Red Majesty, Purple Flora and } \\
\text { Algarve) were exposed to gamma rays treatments }\left(0,25,40,55 \text { and } 70 \text { Gy; Source }{ }^{60} \mathrm{Co}\right) \text {. } \\
\text { These corms were planted under open field condition in Randomized Block Design (RBD) } \\
\text { with factorial concept. The effect of gamma irradiation was studied on various floral } \\
\text { characteristics of the treated plants. The findings indicated that gamma radiation } \\
\text { treatments had significant detrimental effect on days to spike initiation, spike length, rachis } \\
\text { length, number and size of florets, number of days to flowering and flower duration in all } \\
\text { the varieties under study. Plants treated with higher doses ( } 55 \mathrm{~Gy} \text { and } 70 \mathrm{~Gy} \text { ) showed } \\
\text { deleterious effect of ionising radiations although at lowest dose (25 Gy) plants were not } \\
\text { affected much. Plant height was reduced after irradiation as compared to untreated plants } \\
\text { and was recorded minimum at highest doses. Radiation treatments at higher doses caused } \\
\text { delay in spike initiation with decrease in spike length, number and size of florets in vM1. } \\
\text { However, at lower doses diminishing effect of gamma irradiation was observed in vM2. }\end{array}$} \\
\hline & \\
\hline & \\
\hline Arti & \\
\hline $\begin{array}{l}18 \\
:\end{array}$ & \\
\hline
\end{tabular}

\section{Introduction}

Gladiolus (Gladiolus spp.), the queen of bulbous ornamentals, is one of the leading geophytes grown worldwide. It is very popular because of its majestic spikes having florets of dazzling colours. Radiation technology has proven to be useful for mutation breeding and has contributed towards improvements in agricultural crops. Among the mutant varieties, about $90 \%$ were generated by using radiation (Asharaf et al., 2003). X-rays and gamma rays $(\gamma)$ are ionizing radiations and interact with atoms and molecules to produce free radicals in cells. The advantages of physical mutagens, particularly gamma rays are accurate dosimetry, reasonable reproducibility, and high and uniform penetration of multicellular system (Jain, 2005). 


\section{Materials and Methods}

The experiment was conducted at Model Floriculture centre, G. B. Pant University of Agriculture and Technology, Pantnagar. Corms of Gladiolus varieties Yellow Golden, Nathan Red, White Friendship, American Beauty, Red Majesty, Purple Flora and Algarve were irradiated with five different doses of gamma rays viz. 0 (control), 25, 40, 55 and $70 \mathrm{~Gy}$. Irradiation was applied to corms at Punjab Agricultural University, Ludhiana (Low Dose Irradiator 2000 ANSIN4333.1). These irradiated corms were planted in the field within 24 hours of treatment in Factorial RBD concept. Data were recorded in vM1 and vM2 generations on different floral parameters.

\section{Results and Discussion}

Floral characteristics of plants were investigated at flowering stage. Floral characters of gladiolus were significantly affected by different gamma rays doses, varieties and their interactions. The results presented in Table 1,2 and 3 indicate that minimum days to spike initiation (83.48 days), opening of first floret (102.7) and full blooming were taken by untreated plants and it was found that increase in the dose of gamma rays delayed the blooming. Maximum delay was recorded in 70 Gy treated plants in vM1 generation. In vM2, the results altered and spike initiation (73.73 days), opening of first floret (94.65 days) and full blooming (105.77days) was slightly earlier in plants treated with 25 Gy dose as compare to untreated ones. Among varieties, Nathan Red took minimum days to spike initiation (81.01 days) in vM1, whereas in vM2, variety Purple Flora took minimum days for spike initiation (71.09 days) followed by Nathan Red (72.93 days). The interaction effect shows that earliest first floret opening and blooming was recorded in untreated plants of Purple Flora in
vM1, while in 25 Gy treated plants of variety Purple Flora in vM2. Most delayed opening of first floret and blooming was recorded in plants of variety Red Majesty at 70 Gy in vM1 and in variety Yellow Golden at $70 \mathrm{~Gy}$ in vM2.

These results are in close conformity with Srivastava and Singh (2002) who recorded inhibitory effect of 60 and 80 Gy gamma rays irradiation on heading and subsequent flowering of gladiolus. Delay in spike emergence might be due to disturbance in biochemical pathways which assists in flower induction pathway (Bagnall et al., 1995). The delay was less at lowest dose and increased as the dose increased. Similar results were also recorded by Banerji et al., (1981) in Gladiolus psittacinus var. Hookeri cultivars Orange and Red and Kumari et al., (2013) in chrysanthemum. Marked delay in flower organ development in irradiated plants during or immediately after flower differentiation was recorded by Matsubara (1975), while studying the effect of gamma rays on bulbs of tulip at different stages of flower development. Further, it is also evident from the data that per cent delay in flowering was less in vM2 as compare to vM1 which might be due to the diminishing effect of gamma irradiation in vM2 generation and more physiological disturbances in vM1 due to immediate effect of gamma irradiation. These findings are in line with Rather et al., (2002).

Flower size and number of florets was significantly affected by gamma irradiation, varieties and interaction of both. Unirradiated plants had largest flower size and number of florets in vM1 while number of florets was slightly increased as compared to untreated plants in vM2.

Exposure to gamma rays resulted in decrease in size and number of florets leading to minimum at 70 Gy (Table 4-8). 
Table.1 Effect of gamma irradiation on days to spike initiation in different gladiolus varieties

\begin{tabular}{|c|c|c|c|c|c|c|c|c|c|c|c|c|}
\hline \multicolumn{13}{|c|}{ Days to spike initiation } \\
\hline & \multicolumn{6}{|c|}{ vM1 (2012-13) } & \multicolumn{6}{|c|}{ vM2 (2013-14) } \\
\hline & $\begin{array}{l}\mathbf{0 ~ G y} \\
\left(\mathbf{T}_{1}\right)\end{array}$ & $\begin{array}{c}25 \mathbf{G y} \\
\left(\mathbf{T}_{2}\right)\end{array}$ & $\begin{array}{c}40 \mathrm{~Gy} \\
\left(\mathrm{~T}_{3}\right)\end{array}$ & $\begin{array}{c}55 \mathbf{G y} \\
\left(\mathbf{T}_{4}\right)\end{array}$ & $\begin{array}{c}70 \text { Gy } \\
\left(T_{5}\right)\end{array}$ & Mean & $\begin{array}{l}\mathbf{0 ~ G y} \\
\left(\mathbf{T}_{1}\right)\end{array}$ & $\begin{array}{c}25 \mathbf{G y} \\
\left(\mathbf{T}_{2}\right)\end{array}$ & $\begin{array}{c}40 \mathbf{G y} \\
\left(\mathbf{T}_{3}\right)\end{array}$ & $\begin{array}{c}55 \mathbf{G y} \\
\left(\mathbf{T}_{4}\right)\end{array}$ & $\begin{array}{c}70 \mathbf{G y} \\
\left(\mathbf{T}_{5}\right)\end{array}$ & Mean \\
\hline Yellow Golden $\left(\mathbf{V}_{1}\right)$ & 90.53 & $\begin{array}{l}92.00 \\
(1.62)\end{array}$ & $\begin{array}{l}95.08 \\
(5.03)\end{array}$ & $\begin{array}{c}99.83 \\
(10.27) \\
\end{array}$ & $\begin{array}{l}102.33 \\
(13.03)\end{array}$ & 95.96 & 86.43 & $\begin{array}{c}84.97 \\
(-1.69)\end{array}$ & $\begin{array}{l}89.32 \\
(3.34)\end{array}$ & $\begin{array}{l}92.23 \\
(6.71)\end{array}$ & $\begin{array}{l}93.33 \\
(7.98) \\
\end{array}$ & 89.26 \\
\hline Nathan Red ( $\left.\mathbf{V}_{2}\right)$ & 73.75 & $\begin{array}{l}76.68 \\
(3.97)\end{array}$ & $\begin{array}{c}82.77 \\
(12.23)\end{array}$ & $\begin{array}{c}84.83 \\
(15.02)\end{array}$ & $\begin{array}{c}87.00 \\
(17.97)\end{array}$ & 81.01 & 70.60 & $\begin{array}{c}65.33 \\
(-7.46)\end{array}$ & $\begin{array}{l}74.50 \\
(5.52)\end{array}$ & $\begin{array}{l}75.50 \\
(6.94)\end{array}$ & $\begin{array}{c}78.73 \\
(11.52)\end{array}$ & 72.93 \\
\hline $\begin{array}{l}\text { White Friendship } \\
\left(\mathbf{V}_{3}\right)\end{array}$ & 81.10 & $\begin{array}{l}83.83 \\
(3.37)\end{array}$ & $\begin{array}{l}87.40 \\
(7.77)\end{array}$ & $\begin{array}{c}90.17 \\
(11.18)\end{array}$ & $\begin{array}{c}95.92 \\
(18.27)\end{array}$ & 87.68 & 74.33 & $\begin{array}{l}71.57 \\
(-3.71)\end{array}$ & $\begin{array}{l}77.33 \\
(4.04)\end{array}$ & $\begin{array}{l}81.00 \\
(8.97)\end{array}$ & $\begin{array}{c}85.92 \\
(15.59)\end{array}$ & 78.03 \\
\hline Jester Gold $\left(\mathbf{V}_{4}\right)$ & 93.50 & $\begin{array}{l}94.42 \\
(0.98)\end{array}$ & $\begin{array}{l}100.10 \\
(7.06)\end{array}$ & $\begin{array}{l}104.00 \\
(11.23)\end{array}$ & $\begin{array}{l}106.92 \\
(14.35)\end{array}$ & 99.79 & 83.50 & $\begin{array}{c}83.00 \\
(-0.60)\end{array}$ & $\begin{array}{l}87.73 \\
(5.07)\end{array}$ & $\begin{array}{c}92.33 \\
(10.57)\end{array}$ & $\begin{array}{c}95.83 \\
(14.77)\end{array}$ & 88.48 \\
\hline $\begin{array}{l}\text { American Beauty } \\
\left(V_{5}\right)\end{array}$ & 81.67 & $\begin{array}{l}81.80 \\
(0.16)\end{array}$ & $\begin{array}{l}88.90 \\
(8.85)\end{array}$ & $\begin{array}{c}92.27 \\
(12.98)\end{array}$ & $\begin{array}{c}96.17 \\
(17.75)\end{array}$ & 88.16 & 69.17 & $\begin{array}{c}68.17 \\
(-1.45)\end{array}$ & $\begin{array}{l}74.43 \\
(7.60)\end{array}$ & $\begin{array}{c}78.57 \\
(13.59)\end{array}$ & $\begin{array}{c}79.47 \\
(14.89)\end{array}$ & 73.96 \\
\hline Red Majesty $\left(\mathbf{V}_{6}\right)$ & 92.43 & $\begin{array}{c}90.83 \\
(-1.73)\end{array}$ & $\begin{array}{l}96.60 \\
(4.51)\end{array}$ & $\begin{array}{l}102.50 \\
(10.89)\end{array}$ & $\begin{array}{l}105.00 \\
(13.60)\end{array}$ & 97.47 & 81.08 & $\begin{array}{l}81.40 \\
(0.39)\end{array}$ & $\begin{array}{l}84.52 \\
(4.24)\end{array}$ & $\begin{array}{l}88.43 \\
(9.07)\end{array}$ & $\begin{array}{c}92.23 \\
(13.75)\end{array}$ & 85.53 \\
\hline Purple Flora $\left(V_{7}\right)$ & 75.45 & $\begin{array}{r}76.00 \\
(0.73)\end{array}$ & $\begin{array}{l}82.40 \\
(9.21)\end{array}$ & $\begin{array}{c}88.67 \\
(17.52)\end{array}$ & $\begin{array}{c}92.98 \\
(23.23)\end{array}$ & 83.10 & 66.68 & $\begin{array}{c}65.17 \\
(-2.26)\end{array}$ & $\begin{array}{l}70.67 \\
(5.98)\end{array}$ & $\begin{array}{c}74.17 \\
(11.23)\end{array}$ & $\begin{array}{c}78.77 \\
(18.13)\end{array}$ & 71.09 \\
\hline Algarve $\left(V_{8}\right)$ & 79.42 & $\begin{array}{l}83.50 \\
(5.14)\end{array}$ & $\begin{array}{c}90.33 \\
(13.74)\end{array}$ & $\begin{array}{c}93.00 \\
(17.10)\end{array}$ & $\begin{array}{c}98.73 \\
(24.31)\end{array}$ & 89.00 & 73.60 & $\begin{array}{c}70.27 \\
(-4.52)\end{array}$ & $\begin{array}{l}78.00 \\
(5.98)\end{array}$ & $\begin{array}{c}84.83 \\
(15.26)\end{array}$ & $\begin{array}{c}88.50 \\
(20.24)\end{array}$ & 79.04 \\
\hline Mean & 83.48 & 84.88 & 90.45 & 94.41 & 98.13 & & 75.68 & 73.73 & 79.56 & 83.38 & 86.60 & \\
\hline & & & \multicolumn{2}{|c|}{ CD at $5 \%$} & \multicolumn{2}{|c|}{ SEm \pm} & & & \multicolumn{2}{|c|}{ CD at $5 \%$} & \multicolumn{2}{|c|}{ SEm \pm} \\
\hline \multicolumn{3}{|l|}{ Gamma Radiation } & \multicolumn{2}{|c|}{0.97} & \multicolumn{2}{|c|}{0.34} & & & \multicolumn{2}{|c|}{1.13} & \multicolumn{2}{|c|}{0.40} \\
\hline \multicolumn{3}{|l|}{ Varieties } & \multicolumn{2}{|c|}{1.23} & \multicolumn{2}{|c|}{0.44} & & & \multicolumn{2}{|c|}{1.43} & \multicolumn{2}{|c|}{0.51} \\
\hline \multicolumn{3}{|c|}{ Gamma Radiation * Varieties } & \multicolumn{2}{|c|}{2.75} & \multicolumn{2}{|c|}{0.97} & & & \multicolumn{2}{|c|}{3.19} & \multicolumn{2}{|c|}{1.13} \\
\hline
\end{tabular}

*Values in parentheses represent percent deviation from the control, where ( ) represent percent increase and (-) represent percent decrease 
Table.2 Effect of gamma irradiation on days to opening of first floret in different gladiolus varieties

\begin{tabular}{|c|c|c|c|c|c|c|c|c|c|c|c|c|}
\hline \multicolumn{13}{|c|}{ Days to opening of first floret } \\
\hline & \multicolumn{6}{|c|}{ vM1 (2012-13) } & \multicolumn{6}{|c|}{ vM2 (2013-14) } \\
\hline & $\begin{array}{l}\mathbf{0} \mathbf{G y} \\
\left(\mathrm{T}_{1}\right)\end{array}$ & $\begin{array}{c}25 \mathbf{G y} \\
\left(\mathrm{T}_{2}\right)\end{array}$ & $\begin{array}{c}40 \mathbf{G y} \\
\left(\mathbf{T}_{3}\right)\end{array}$ & $\begin{array}{c}55 \mathbf{G y} \\
\left(\mathrm{T}_{4}\right)\end{array}$ & $\begin{array}{l}70 \mathbf{G y} \\
\left(\mathbf{T}_{5}\right)\end{array}$ & Mean & $\begin{array}{l}\mathbf{0 ~ G y} \\
\left(\mathbf{T}_{1}\right)\end{array}$ & $\begin{array}{c}25 \mathbf{G y} \\
\left(\mathrm{T}_{2}\right)\end{array}$ & $\begin{array}{c}40 \mathrm{~Gy} \\
\left(\mathbf{T}_{3}\right)\end{array}$ & $\begin{array}{c}55 \mathbf{G y} \\
\left(\mathrm{T}_{4}\right)\end{array}$ & $\begin{array}{c}70 \mathbf{~ G y} \\
\left(\mathbf{T}_{5}\right)\end{array}$ & Mean \\
\hline Yellow Golden $\left(\mathbf{V}_{1}\right)$ & 105.00 & $\begin{array}{l}110.23 \\
(4.98)\end{array}$ & $\begin{array}{l}115.23 \\
(9.74)\end{array}$ & $\begin{array}{l}121.10 \\
(15.33)\end{array}$ & $\begin{array}{l}127.40 \\
(21.33)\end{array}$ & 115.79 & 104.60 & $\begin{array}{l}102.27 \\
(-2.23)\end{array}$ & $\begin{array}{l}108.00 \\
(3.25)\end{array}$ & $\begin{array}{l}112.20 \\
(7.27)\end{array}$ & $\begin{array}{l}118.43 \\
(13.22)\end{array}$ & 109.10 \\
\hline Nathan Red $\left(\mathbf{V}_{2}\right)$ & 95.77 & $\begin{array}{l}100.73 \\
(5.18)\end{array}$ & $\begin{array}{l}106.10 \\
(10.79)\end{array}$ & $\begin{array}{l}109.53 \\
(14.37)\end{array}$ & $\begin{array}{l}113.47 \\
(18.48)\end{array}$ & 105.12 & 92.60 & $\begin{array}{c}87.25 \\
(-5.78)\end{array}$ & $\begin{array}{l}96.60 \\
(4.32)\end{array}$ & $\begin{array}{l}98.53 \\
(6.40)\end{array}$ & $\begin{array}{l}104.87 \\
(13.25)\end{array}$ & 95.97 \\
\hline $\begin{array}{l}\text { White Friendship } \\
\left(\mathbf{V}_{3}\right)\end{array}$ & 100.30 & $\begin{array}{l}102.10 \\
(1.79)\end{array}$ & $\begin{array}{l}109.83 \\
(9.50)\end{array}$ & $\begin{array}{l}115.33 \\
(14.99)\end{array}$ & $\begin{array}{l}122.33 \\
(21.96)\end{array}$ & 109.98 & 95.00 & $\begin{array}{c}90.57 \\
(-4.66)\end{array}$ & $\begin{array}{l}100.17 \\
(5.44)\end{array}$ & $\begin{array}{l}106.10 \\
(11.68)\end{array}$ & $\begin{array}{l}110.67 \\
(16.49)\end{array}$ & 100.50 \\
\hline Jester Gold $\left(\mathbf{V}_{4}\right)$ & 109.20 & $\begin{array}{l}116.10 \\
(6.32)\end{array}$ & $\begin{array}{l}123.33 \\
(12.94)\end{array}$ & $\begin{array}{l}125.33 \\
(14.77)\end{array}$ & $\begin{array}{l}129.27 \\
(18.38)\end{array}$ & 120.65 & 104.00 & $\begin{array}{l}106.27 \\
(2.18)\end{array}$ & $\begin{array}{l}112.83 \\
(8.49)\end{array}$ & $\begin{array}{l}114.27 \\
(9.88)\end{array}$ & $\begin{array}{l}117.27 \\
(12.76)\end{array}$ & 110.93 \\
\hline $\begin{array}{l}\text { American Beauty } \\
\left(V_{5}\right)\end{array}$ & 103.17 & $\begin{array}{l}102.80 \\
(-0.36)\end{array}$ & $\begin{array}{l}111.67 \\
(8.24)\end{array}$ & $\begin{array}{l}117.00 \\
(13.41)\end{array}$ & $\begin{array}{l}122.33 \\
(18.57)\end{array}$ & 111.39 & 89.92 & $\begin{array}{c}89.63 \\
(-0.32)\end{array}$ & $\begin{array}{l}97.07 \\
(7.95)\end{array}$ & $\begin{array}{l}102.47 \\
(13.96)\end{array}$ & $\begin{array}{l}105.77 \\
(17.63)\end{array}$ & 96.97 \\
\hline Red Majesty $\left(V_{6}\right)$ & 112.73 & $\begin{array}{l}114.17 \\
(1.28)\end{array}$ & $\begin{array}{l}122.60 \\
(8.76)\end{array}$ & $\begin{array}{l}128.33 \\
(13.84)\end{array}$ & $\begin{array}{l}132.93 \\
(17.92)\end{array}$ & 122.15 & 103.87 & $\begin{array}{l}102.93 \\
(-0.90)\end{array}$ & $\begin{array}{l}109.83 \\
(5.74)\end{array}$ & $\begin{array}{l}115.93 \\
(11.61)\end{array}$ & $\begin{array}{l}116.73 \\
(12.38)\end{array}$ & 109.86 \\
\hline Purple Flora $\left(V_{7}\right)$ & 94.33 & $\begin{array}{l}95.00 \\
(0.71)\end{array}$ & $\begin{array}{l}99.67 \\
(5.66)\end{array}$ & $\begin{array}{l}107.17 \\
(13.61)\end{array}$ & $\begin{array}{l}118.33 \\
(25.44)\end{array}$ & 102.90 & 86.83 & $\begin{array}{c}84.57 \\
(-2.60)\end{array}$ & $\begin{array}{l}92.67 \\
(6.73)\end{array}$ & $\begin{array}{c}97.33 \\
(12.09)\end{array}$ & $\begin{array}{l}105.00 \\
(20.93)\end{array}$ & 93.28 \\
\hline Algarve $\left(\mathbf{V}_{8}\right)$ & 101.33 & $\begin{array}{l}106.50 \\
(5.10)\end{array}$ & $\begin{array}{l}115.00 \\
(13.49)\end{array}$ & $\begin{array}{l}119.67 \\
(18.10)\end{array}$ & $\begin{array}{l}122.67 \\
(21.06)\end{array}$ & 113.03 & 98.73 & $\begin{array}{c}93.70 \\
(-5.09)\end{array}$ & $\begin{array}{l}104.00 \\
(5.34)\end{array}$ & $\begin{array}{l}111.80 \\
(13.24)\end{array}$ & $\begin{array}{l}116.53 \\
(18.03)\end{array}$ & 104.95 \\
\hline Mean & 102.73 & 105.95 & 112.93 & 117.93 & 123.59 & & 96.94 & 94.65 & 102.65 & 107.33 & 111.91 & \\
\hline & & & \multicolumn{2}{|c|}{ CD at $5 \%$} & \multicolumn{2}{|c|}{ SEm \pm} & & & \multicolumn{2}{|c|}{ CD at $5 \%$} & \multicolumn{2}{|c|}{ SEm \pm} \\
\hline \multicolumn{3}{|l|}{ Gamma Radiation } & \multicolumn{2}{|c|}{1.01} & \multicolumn{2}{|c|}{0.36} & & & \multicolumn{2}{|c|}{1.14} & \multicolumn{2}{|c|}{0.40} \\
\hline \multicolumn{3}{|l|}{ Varieties } & \multicolumn{2}{|c|}{1.28} & \multicolumn{2}{|c|}{0.45} & & & \multicolumn{2}{|c|}{1.44} & \multicolumn{2}{|c|}{0.51} \\
\hline \multicolumn{3}{|c|}{ Gamma Radiation * Varieties } & \multicolumn{2}{|c|}{2.86} & \multicolumn{2}{|c|}{1.02} & & & \multicolumn{2}{|c|}{3.22} & \multicolumn{2}{|c|}{1.14} \\
\hline
\end{tabular}

*Values in parentheses represent percent deviation from the control, where ( ) represent percent increase and (-) represent percent decrease 
Table.3 Effect of gamma irradiation on basal floret diameter $(\mathrm{cm})$ in different gladiolus varieties

\begin{tabular}{|c|c|c|c|c|c|c|c|c|c|c|c|c|}
\hline \multicolumn{13}{|c|}{ Floret diameter $(\mathrm{cm})$} \\
\hline & \multicolumn{6}{|c|}{ vM1 (2012-13) } & \multicolumn{6}{|c|}{ vM2 (2013-14) } \\
\hline & $\begin{array}{c}\mathbf{0} \mathbf{G y} \\
\left(\mathbf{T}_{1}\right)\end{array}$ & $\begin{array}{c}25 \text { Gy } \\
\left(T_{2}\right)\end{array}$ & $\begin{array}{c}40 \mathbf{~ G y} \\
\left(\mathbf{T}_{\mathbf{3}}\right)\end{array}$ & $\begin{array}{c}55 \mathbf{G y} \\
\left(\mathbf{T}_{4}\right)\end{array}$ & $\begin{array}{c}70 \mathbf{G y} \\
\left(\mathbf{T}_{5}\right)\end{array}$ & Mean & $\begin{array}{c}\mathbf{0} \mathbf{G y} \\
\left(\mathbf{T}_{1}\right)\end{array}$ & $\begin{array}{c}25 \text { Gy } \\
\left(T_{2}\right)\end{array}$ & $\begin{array}{c}40 \mathrm{~Gy} \\
\left(\mathrm{~T}_{3}\right)\end{array}$ & $\begin{array}{c}55 \mathbf{~ G y} \\
\left(\mathbf{T}_{4}\right)\end{array}$ & $\begin{array}{c}70 \mathbf{~ G y} \\
\left(\mathbf{T}_{5}\right)\end{array}$ & Mean \\
\hline \multirow[t]{2}{*}{ Yellow Golden $\left(V_{1}\right)$} & 10.53 & 10.22 & 9.72 & 9.25 & 8.57 & 9.66 & 10.75 & 10.52 & 9.90 & 9.00 & 8.42 & 9.72 \\
\hline & & $(-2.94)$ & $(-7.69)$ & $\begin{array}{c}(- \\
12.16)\end{array}$ & $(-18.61)$ & & & $(-2.14)$ & $(-7.91)$ & $(-16.28)$ & $(-21.67)$ & \\
\hline \multirow[t]{2}{*}{ Nathan Red $\left(\mathbf{V}_{2}\right)$} & 9.07 & 8.98 & 8.71 & 8.42 & 7.58 & 8.55 & 8.63 & 8.99 & 8.65 & 8.33 & 7.43 & 8.41 \\
\hline & & $(-0.99)$ & $(-3.97)$ & $(-7.17)$ & $(-16.43)$ & & & $(4.17)$ & $(0.23)$ & $(-3.48)$ & $(-13.90)$ & \\
\hline \multirow{2}{*}{$\begin{array}{l}\text { White Friendship } \\
\left(\mathbf{V}_{\mathbf{3}}\right)\end{array}$} & 11.00 & 10.70 & 10.12 & 9.29 & 7.92 & 9.81 & 10.83 & 10.63 & 10.24 & 9.47 & 8.13 & 9.86 \\
\hline & & $(-2.73)$ & $(-8.00)$ & $\begin{array}{c}(- \\
15.55)\end{array}$ & $(-28.00)$ & & & $(-1.85)$ & $(-5.45)$ & $(-12.56)$ & $(-24.93)$ & \\
\hline \multirow[t]{2}{*}{ Jester Gold $\left(V_{4}\right)$} & 9.82 & 9.60 & 9.49 & 9.16 & 8.28 & 9.27 & 10.03 & 9.64 & 9.52 & 9.22 & 8.63 & 9.41 \\
\hline & & $(-2.24)$ & $(-3.36)$ & $(-6.72)$ & $(-15.68)$ & & & $(-3.89)$ & $(-5.08)$ & $(-8.08)$ & $(-13.96)$ & \\
\hline \multirow{2}{*}{$\begin{array}{l}\text { American Beauty } \\
\left(V_{5}\right)\end{array}$} & 10.21 & 9.77 & 9.12 & 8.66 & 7.95 & 9.14 & 10.17 & 9.52 & 9.03 & 8.35 & 8.03 & 9.02 \\
\hline & & $(-4.31)$ & $\begin{array}{c}(- \\
10.68)\end{array}$ & $\begin{array}{c}(- \\
15.18)\end{array}$ & $(-22.14)$ & & & $(-6.39)$ & $\begin{array}{c}(- \\
11.21)\end{array}$ & $(-17.90)$ & $(-21.04)$ & \\
\hline \multirow[t]{2}{*}{ Red Majesty $\left(V_{6}\right)$} & 10.30 & 10.20 & 9.78 & 9.38 & 8.90 & 9.71 & 10.47 & 10.42 & 9.73 & 9.54 & 8.83 & 9.80 \\
\hline & & $(-0.97)$ & $(-5.05)$ & $(-8.93)$ & $(-13.59)$ & & & $(-0.48)$ & $(-7.07)$ & $(-8.88)$ & $(-15.66)$ & \\
\hline \multirow[t]{2}{*}{ Purple Flora $\left(\mathbf{V}_{7}\right)$} & 9.44 & 9.31 & 8.97 & 7.21 & 6.73 & 8.33 & 9.55 & 9.37 & 8.80 & 7.42 & 6.78 & 8.39 \\
\hline & & $(-1.38)$ & $(-4.98)$ & $\begin{array}{c}(- \\
23.62)\end{array}$ & $(-28.71)$ & & & $(-1.88)$ & $(-7.85)$ & $(-22.30)$ & $(-29.01)$ & \\
\hline \multirow[t]{2}{*}{ Algarve $\left(\mathbf{V}_{8}\right)$} & 9.32 & 8.77 & 8.49 & 8.23 & 7.51 & 8.46 & 9.29 & 9.00 & 8.67 & 8.41 & 7.28 & 8.53 \\
\hline & & $(-5.90)$ & $(-8.91)$ & $\begin{array}{c}(- \\
11.70)\end{array}$ & $(-19.42)$ & & & $(-3.12)$ & $(-6.67)$ & $(-9.47)$ & $(-21.64)$ & \\
\hline Mean & 9.96 & 9.69 & 9.30 & 8.70 & 7.93 & & 9.97 & 9.76 & 9.32 & 8.72 & 7.94 & \\
\hline
\end{tabular}

*Values in parentheses represent percent deviation from the control, where ( ) represent percent increase and (-) represent percent decrease 
Table.4 Effect of gamma irradiation on number of florets per spike in different gladiolus varieties

\begin{tabular}{|c|c|c|c|c|c|c|c|c|c|c|c|c|}
\hline \multicolumn{13}{|c|}{ Florets per spike } \\
\hline & \multicolumn{6}{|c|}{ vM1 (2012-13) } & \multicolumn{6}{|c|}{ vM2 (2013-14) } \\
\hline & $\begin{array}{c}\mathbf{0} \mathbf{G y} \\
\left(\mathbf{T}_{1}\right)\end{array}$ & $\begin{array}{c}25 \mathbf{G y} \\
\left(\mathbf{T}_{2}\right)\end{array}$ & $\begin{array}{c}40 \mathrm{~Gy} \\
\left(\mathrm{~T}_{3}\right)\end{array}$ & $\begin{array}{c}55 \mathrm{~Gy} \\
\left(\mathrm{~T}_{4}\right)\end{array}$ & $\begin{array}{c}70 \text { Gy } \\
\left(\mathbf{T}_{5}\right)\end{array}$ & Mean & $\begin{array}{c}\mathbf{0} \mathbf{G y} \\
\left(\mathbf{T}_{1}\right)\end{array}$ & $\begin{array}{c}25 \mathbf{G y} \\
\left(\mathbf{T}_{2}\right)\end{array}$ & $\begin{array}{c}40 \mathrm{~Gy} \\
\left(\mathrm{~T}_{3}\right)\end{array}$ & $\begin{array}{c}55 \mathbf{G y} \\
\left(\mathbf{T}_{4}\right)\end{array}$ & $\begin{array}{c}70 \text { Gy } \\
\left(\mathbf{T}_{5}\right)\end{array}$ & Mean \\
\hline \multirow{2}{*}{ Yellow Golden $\left(V_{1}\right)$} & 14.77 & 13.90 & 11.38 & 9.00 & 7.68 & 11.35 & 14.75 & 15.93 & 12.50 & 9.85 & 8.41 & 12.29 \\
\hline & & $(-5.89)$ & $(-22.9) 5$ & $(-39.07)$ & $(-48.00)$ & & & $(8.00)$ & $(-15.25)$ & $(-33.22)$ & $(-42.98$ & \\
\hline \multirow[t]{2}{*}{ Nathan Red ( $\left.\mathbf{V}_{2}\right)$} & 13.83 & 13.40 & 11.20 & 8.57 & 8.18 & 11.04 & 13.33 & 14.12 & 12.03 & 9.05 & 8.10 & 11.33 \\
\hline & & $(-3.11)$ & $(-19.02$ & $(-38.03)$ & $(-40.85$ & & & $(5.93)$ & $(-9.75)$ & $(-32.11)$ & $(-39.23)$ & \\
\hline \multirow[t]{2}{*}{ White Friendship ( $\left.V_{3}\right)$} & 14.10 & 13.43 & 11.31 & 7.12 & 6.73 & 10.54 & 14.27 & 15.07 & 13.17 & 8.12 & 7.43 & 11.61 \\
\hline & & $(-4.75)$ & $(-19.79)$ & $(-49.50)$ & $(-52.27)$ & & & $(5.61)$ & $(-7.71)$ & $(-43.10)$ & $(-47.93)$ & \\
\hline \multirow[t]{2}{*}{ Jester Gold ( $\left.\mathbf{V}_{4}\right)$} & 13.98 & 13.60 & 11.60 & 8.65 & 7.33 & 11.03 & 13.70 & 14.10 & 11.13 & 9.42 & 7.67 & 11.20 \\
\hline & & $(-2.72$ & $(-17.02)$ & $(-38.13)$ & $(-47.57)$ & & & $(2.92)$ & $(-18.76)$ & $(-31.24)$ & $(-44.01)$ & \\
\hline \multirow[t]{2}{*}{ American Beauty $\left(V_{5}\right)$} & 13.20 & 13.45 & 11.15 & 8.97 & 7.85 & 10.92 & 14.05 & 14.40 & 11.87 & 9.52 & 8.90 & 11.75 \\
\hline & & $(1.89$ & $(-15.53)$ & $(-32.05)$ & $(-40.53)$ & & & $(2.49)$ & $(-15.52)$ & $(-32.24)$ & $(-36.65)$ & \\
\hline \multirow[t]{2}{*}{ Red Majesty $\left(V_{6}\right)$} & 14.00 & 14.73 & 11.43 & 6.65 & 5.57 & 10.48 & 14.77 & 15.53 & 12.42 & 7.50 & 6.80 & 11.40 \\
\hline & & $(5.21)$ & $(-18.36)$ & $(-52.50)$ & $(-60.21)$ & & & $(5.15)$ & $(-15.91)$ & $(-49.22)$ & $(-53.96)$ & \\
\hline \multirow[t]{2}{*}{ Purple Flora $\left(V_{7}\right)$} & 13.77 & 13.25 & 10.32 & 5.85 & 4.43 & 9.52 & 13.88 & 14.23 & 12.17 & 7.22 & 6.27 & 10.75 \\
\hline & & $(-3.78)$ & $(-25.05)$ & $(-57.52)$ & $(-67.83)$ & & & $(2.52)$ & $(-12.32)$ & $(-47.98)$ & $(-54.83)$ & \\
\hline \multirow[t]{2}{*}{$\operatorname{Algarve}\left(\mathbf{V}_{8}\right)$} & 12.53 & 12.73 & 9.40 & 7.30 & 5.00 & 9.39 & 13.37 & 14.48 & 10.71 & 8.94 & 6.58 & 10.82 \\
\hline & & $(1.60)$ & $(-24.98)$ & $(-41.74)$ & $(-60.10)$ & & & $(8.30)$ & $(-19.90)$ & $(-33.13)$ & $(-50.79)$ & \\
\hline Mean & 13.77 & 13.56 & 10.97 & 7.76 & 6.60 & & 14.02 & 14.73 & 12.00 & 8.70 & 7.52 & \\
\hline & & & \multicolumn{2}{|c|}{ CD at $5 \%$} & \multicolumn{2}{|l|}{ SEm \pm} & & & \multicolumn{2}{|c|}{ CD at $5 \%$} & \multicolumn{2}{|c|}{ SEm \pm} \\
\hline \multicolumn{3}{|l|}{ Gamma Radiation } & \multicolumn{2}{|c|}{0.32} & \multicolumn{2}{|l|}{0.11} & & & \multicolumn{2}{|c|}{0.35} & \multicolumn{2}{|c|}{0.13} \\
\hline & & & \multicolumn{2}{|c|}{0.40} & \multicolumn{2}{|l|}{0.14} & & & \multicolumn{2}{|c|}{0.44} & \multicolumn{2}{|c|}{0.16} \\
\hline \multicolumn{3}{|l|}{ Gamma Radiation * V } & \multicolumn{2}{|c|}{0.89} & \multicolumn{2}{|l|}{0.31} & & & \multicolumn{2}{|c|}{1.00} & \multicolumn{2}{|c|}{0.35} \\
\hline
\end{tabular}

*Values in parentheses represent percent deviation from the control, where ( ) represent percent increase and (-) represent percent decrease 
Table.5 Effect of gamma irradiation on spike length $(\mathrm{cm})$ in different gladiolus varieties

\begin{tabular}{|c|c|c|c|c|c|c|c|c|c|c|c|c|}
\hline \multicolumn{13}{|c|}{ Spike length (cm) } \\
\hline & \multicolumn{6}{|c|}{ vM1 (2012-13) } & \multicolumn{6}{|c|}{ vM2 (2013-14) } \\
\hline & $\begin{array}{c}\mathbf{0} \mathbf{G y} \\
\left(\mathbf{T}_{1}\right)\end{array}$ & $\begin{array}{c}25 \mathbf{G y} \\
\left(\mathbf{T}_{2}\right)\end{array}$ & $\begin{array}{c}40 \mathrm{~Gy} \\
\left(\mathbf{T}_{3}\right)\end{array}$ & $\begin{array}{c}55 \text { Gy } \\
\left(\mathbf{T}_{4}\right)\end{array}$ & $\begin{array}{c}70 \text { Gy } \\
\left(T_{5}\right)\end{array}$ & Mean & $\begin{array}{c}\mathbf{0} \mathbf{G y} \\
\left(\mathbf{T}_{1}\right)\end{array}$ & $\begin{array}{c}25 \mathbf{G y} \\
\left(\mathbf{T}_{2}\right)\end{array}$ & $\begin{array}{c}40 \mathrm{~Gy} \\
\left(\mathrm{~T}_{3}\right)\end{array}$ & $\begin{array}{c}55 \text { Gy } \\
\left(\mathbf{T}_{4}\right)\end{array}$ & $\begin{array}{c}70 \text { Gy } \\
\left(T_{5}\right)\end{array}$ & Mean \\
\hline \multirow[t]{2}{*}{ Yellow Golden $\left(V_{1}\right)$} & 110.63 & 109.30 & 87.93 & 70.58 & 61.90 & 88.07 & 110.40 & 113.93 & 91.30 & 73.97 & 63.13 & 90.55 \\
\hline & & $(-1.20)$ & $(-20.52)$ & $(-36.20)$ & $(-44.05)$ & & & $(3.20)$ & $(-17.30)$ & $(-33.00)$ & $(-42.82$ & \\
\hline \multirow[t]{2}{*}{ Nathan Red $\left(V_{2}\right)$} & 98.00 & 91.32 & 88.53 & 72.37 & 65.43 & 83.13 & 92.47 & 96.73 & 90.20 & 74.30 & 66.47 & 84.03 \\
\hline & & $(-6.82)$ & $(-9.66)$ & $(-26.15)$ & $(-33.23)$ & & & $(4.61)$ & $(-2.45)$ & $(-19.65)$ & $(-28.12)$ & \\
\hline \multirow[t]{2}{*}{ White Friendship $\left(\mathbf{V}_{3}\right)$} & 99.20 & 97.85 & 80.77 & 51.63 & 43.40 & 74.57 & 100.13 & 102.50 & 84.73 & 57.97 & 45.47 & 78.16 \\
\hline & & $(-1.36)$ & $(-18.58)$ & $(-47.95)$ & $(-56.25)$ & & & $(2.37)$ & $(-15.38)$ & $(-42.11)$ & $(-54.59)$ & \\
\hline \multirow[t]{2}{*}{ Jester Gold $\left(\mathbf{V}_{4}\right)$} & 99.17 & 96.05 & 83.40 & 68.33 & 51.97 & 79.78 & 96.60 & 100.70 & 87.73 & 70.28 & 55.83 & 82.23 \\
\hline & & $(-3.15)$ & $(-15.90)$ & $(-31.10)$ & $(-47.60)$ & & & $(4.24)$ & $(-9.18)$ & $(-27.25)$ & $(-42.20)$ & \\
\hline \multirow[t]{2}{*}{ American Beauty $\left(V_{5}\right)$} & 101.35 & 103.17 & 84.93 & 66.33 & 54.60 & 82.08 & 102.40 & 106.23 & 89.50 & 70.77 & 58.47 & 85.47 \\
\hline & & $(1.80)$ & $(-16.20)$ & $(-34.55)$ & $(-46.13)$ & & & $(3.74)$ & $(-12.60)$ & $(-30.89)$ & $(-42.90)$ & \\
\hline \multirow[t]{2}{*}{ Red Majesty $\left(V_{6}\right)$} & 106.73 & 102.67 & 85.50 & 64.07 & 48.83 & 81.56 & 108.23 & 107.03 & 89.80 & 67.23 & 51.17 & 84.69 \\
\hline & & $(-3.80)$ & $(-19.89)$ & $(-39.97)$ & $(-54.25)$ & & & $(-1.11)$ & $(-17.03)$ & $(-37.88)$ & $(-52.72)$ & \\
\hline \multirow[t]{2}{*}{ Purple Flora $\left(\mathbf{V}_{7}\right)$} & 87.60 & 84.73 & 71.88 & 57.88 & 40.83 & 68.59 & 86.77 & 90.10 & 75.33 & 61.67 & 46.77 & 72.13 \\
\hline & & $(-3.28)$ & $(-17.95)$ & $(-33.93)$ & $(-53.39)$ & & & $(3.84)$ & $(-13.18)$ & $(-28.93)$ & $(-46.10)$ & \\
\hline \multirow[t]{2}{*}{ Algarve $\left(V_{8}\right)$} & 90.67 & 87.50 & 73.45 & 62.22 & 47.73 & 72.31 & 89.03 & 92.77 & 77.83 & 70.38 & 66.08 & 79.22 \\
\hline & & $(-3.50)$ & $(-18.99)$ & $(-31.38)$ & $(-47.36)$ & & & $(4.20)$ & $(-12.58)$ & $(-20.95)$ & $(-25.78)$ & \\
\hline Mean & 99.17 & 96.57 & 82.05 & 64.18 & 51.84 & & 98.25 & 101.25 & 85.8 & 68.32 & 56.67 & \\
\hline & & & \multicolumn{2}{|c|}{ CD at $5 \%$} & \multicolumn{2}{|c|}{ SEm \pm} & & & \multicolumn{2}{|c|}{ CD at $5 \%$} & \multicolumn{2}{|c|}{ SEm \pm} \\
\hline \multicolumn{3}{|l|}{ Gamma Radiation } & \multicolumn{2}{|c|}{1.90} & \multicolumn{2}{|c|}{0.67} & & & \multicolumn{2}{|c|}{2.15} & \multicolumn{2}{|c|}{0.76} \\
\hline Varieties & & & \multicolumn{2}{|c|}{2.41} & \multicolumn{2}{|c|}{0.85} & & & \multicolumn{2}{|c|}{2.72} & \multicolumn{2}{|c|}{0.97} \\
\hline \multicolumn{3}{|c|}{ Gamma Radiation * Varieties } & \multicolumn{2}{|c|}{5.38} & \multicolumn{2}{|c|}{1.91} & & & \multicolumn{2}{|c|}{6.09} & \multicolumn{2}{|c|}{2.16} \\
\hline
\end{tabular}

*Values in parentheses represent percent deviation from the control, where ( ) represent percent increase and (-) represent percent decrease 
Table.6 Effect of gamma irradiation on rachis length $(\mathrm{cm})$ in different gladiolus varieties

\begin{tabular}{|c|c|c|c|c|c|c|c|c|c|c|c|c|}
\hline \multicolumn{13}{|c|}{ Rachis length (cm) } \\
\hline & \multicolumn{6}{|c|}{ vM1 (2012-13) } & \multicolumn{6}{|c|}{ vM2 (2013-14) } \\
\hline & $\begin{array}{l}\mathbf{0} \mathbf{G y} \\
\left(\mathbf{T}_{1}\right)\end{array}$ & $\begin{array}{c}25 \mathbf{G y} \\
\left(\mathbf{T}_{2}\right)\end{array}$ & $\begin{array}{c}40 \mathrm{~Gy} \\
\left(\mathrm{~T}_{3}\right)\end{array}$ & $\begin{array}{c}55 \mathbf{G y} \\
\left(\mathbf{T}_{4}\right)\end{array}$ & $\begin{array}{c}70 \text { Gy } \\
\left(\mathbf{T}_{5}\right)\end{array}$ & Mean & $\begin{array}{c}\mathbf{0} \mathbf{G y} \\
\left(\mathbf{T}_{1}\right)\end{array}$ & $\begin{array}{c}25 \mathbf{G y} \\
\left(\mathrm{T}_{2}\right)\end{array}$ & $\begin{array}{c}40 \mathrm{~Gy} \\
\left(\mathrm{~T}_{3}\right)\end{array}$ & $\begin{array}{c}55 \mathbf{G y} \\
\left(\mathbf{T}_{4}\right)\end{array}$ & $\begin{array}{c}70 \text { Gy } \\
\left(\mathbf{T}_{5}\right)\end{array}$ & Mean \\
\hline \multirow[t]{2}{*}{ Yellow Golden $\left(V_{1}\right)$} & 64.40 & 59.00 & 51.88 & 40.24 & 28.90 & 48.89 & 60.30 & 64.83 & 55.30 & 42.77 & 30.47 & 50.73 \\
\hline & & $(-8.39)$ & $(-19.44)$ & $(-37.52)$ & $(-55.12$ & & & $(7.51)$ & $(-8.29)$ & $(-29.07)$ & $(-49.47$ & \\
\hline \multirow[t]{2}{*}{ Nathan Red ( $\left.\mathbf{V}_{2}\right)$} & 60.47 & 54.60 & 51.35 & 41.82 & 37.02 & 49.05 & 55.83 & 61.23 & 53.62 & 43.78 & 40.00 & 50.89 \\
\hline & & $(-9.71)$ & $(-15.08)$ & $(-30.84)$ & $(-38.78)$ & & & $(9.67)$ & $(-3.96)$ & $(-21.58)$ & $(-28.35)$ & \\
\hline \multirow[t]{2}{*}{ White Friendship $\left(\mathbf{V}_{3}\right)$} & 58.13 & 55.78 & 43.50 & 29.00 & 15.75 & 40.43 & 57.13 & 59.33 & 47.63 & 31.53 & 17.78 & 42.68 \\
\hline & & $(-4.04)$ & $(-25.17)$ & $(-50.11)$ & $(-72.91)$ & & & $(3.85)$ & $(-16.63)$ & $(-44.81)$ & $(-68.88)$ & \\
\hline \multirow[t]{2}{*}{ Jester Gold ( $\left.\mathbf{V}_{4}\right)$} & 55.67 & 51.05 & 47.60 & 32.12 & 23.73 & 42.03 & 51.55 & 56.67 & 50.05 & 34.00 & 26.43 & 43.74 \\
\hline & & $(-8.30)$ & $(-14.50)$ & $(-42.30)$ & $(-57.37)$ & & & $(9.93)$ & $(-2.91)$ & $(-34.04)$ & $(-48.73)$ & \\
\hline \multirow[t]{2}{*}{ American Beauty ( $\left.V_{5}\right)$} & 55.26 & 57.30 & 50.45 & 37.82 & 26.92 & 45.55 & 54.93 & 60.33 & 51.63 & 38.40 & 27.60 & 46.58 \\
\hline & & $(3.69)$ & $(-8.70)$ & $(-31.56)$ & $(-51.28)$ & & & $(9.83)$ & $(-6.01)$ & $(-30.09)$ & $(-49.75)$ & \\
\hline \multirow[t]{2}{*}{ Red Majesty $\left(V_{6}\right)$} & 58.50 & 56.10 & 41.47 & 28.53 & 20.30 & 40.98 & 56.80 & 59.33 & 44.20 & 30.20 & 22.27 & 42.56 \\
\hline & & $(-4.10)$ & $(-29.11)$ & $(-51.23)$ & $(-65.30)$ & & & $(4.45)$ & $(-22.18)$ & $(-46.83)$ & $(-60.79)$ & \\
\hline \multirow[t]{2}{*}{ Purple Flora $\left(V_{7}\right)$} & 49.83 & 48.03 & 40.05 & 28.40 & 19.47 & 37.16 & 47.93 & 52.25 & 43.03 & 28.53 & 22.05 & 38.76 \\
\hline & & $(-3.61)$ & $(-19.63)$ & $(-43.01$ & $(-60.93)$ & & & $(9.01)$ & $(-10.22)$ & $(-40.48)$ & $(-54.00)$ & \\
\hline \multirow[t]{2}{*}{ Algarve $\left(V_{8}\right)$} & 57.83 & 53.44 & 45.37 & 33.50 & 26.28 & 43.29 & 54.12 & 58.83 & 48.33 & 36.20 & 29.63 & 45.42 \\
\hline & & $(-7.59)$ & $(-21.55)$ & $(-42.07)$ & $(-54.56)$ & & & $(8.70)$ & $(-10.70)$ & $(-33.11)$ & $(-45.25)$ & \\
\hline Mean & 57.51 & 54.41 & 46.46 & 33.93 & 24.8 & & 54.83 & 59.1 & 49.23 & 35.68 & 27.03 & \\
\hline & & & \multicolumn{2}{|c|}{ CD at $5 \%$} & \multicolumn{2}{|c|}{ SEm \pm} & & & \multicolumn{2}{|c|}{ CD at $5 \%$} & \multicolumn{2}{|c|}{ SEm \pm} \\
\hline \multicolumn{3}{|l|}{ Gamma Radiation } & \multicolumn{2}{|c|}{0.90} & \multicolumn{2}{|c|}{0.32} & & & \multicolumn{2}{|c|}{1.15} & \multicolumn{2}{|c|}{0.41} \\
\hline \multicolumn{3}{|l|}{ Varieties } & \multicolumn{2}{|c|}{1.14} & \multicolumn{2}{|c|}{0.40} & & & \multicolumn{2}{|c|}{1.46} & \multicolumn{2}{|c|}{0.52} \\
\hline \multicolumn{3}{|c|}{ Gamma Radiation * Varieties } & \multicolumn{2}{|c|}{2.54} & \multicolumn{2}{|c|}{0.90} & & & \multicolumn{2}{|c|}{3.26} & \multicolumn{2}{|c|}{1.15} \\
\hline
\end{tabular}

Values in parentheses represent percent deviation from the control, where ( ) represent percent increase and (-) represent percent decrease 
Table.7 Effect of gamma irradiation on days to full bloom in different gladiolus varieties

\begin{tabular}{|c|c|c|c|c|c|c|c|c|c|c|c|c|}
\hline \multicolumn{13}{|c|}{ Days to full bloom } \\
\hline & \multicolumn{6}{|c|}{ vM1 (2012-13) } & \multicolumn{6}{|c|}{ vM2 (2013-14) } \\
\hline & $\begin{array}{c}\text { 0 Gy } \\
\left(\mathbf{T}_{1}\right)\end{array}$ & $\begin{array}{c}25 \mathbf{G y} \\
\left(\mathbf{T}_{2}\right)\end{array}$ & $\begin{array}{c}\mathbf{4 0} \mathbf{~ G y} \\
\left(\mathbf{T}_{\mathbf{3}}\right)\end{array}$ & $\begin{array}{c}55 \text { Gy } \\
\left(\mathbf{T}_{4}\right)\end{array}$ & $\begin{array}{c}70 \text { Gy } \\
\left(\mathbf{T}_{5}\right)\end{array}$ & Mean & $\begin{array}{c}\mathbf{0} \text { Gy } \\
\left(\mathbf{T}_{1}\right)\end{array}$ & $\begin{array}{c}25 \mathbf{G y} \\
\left(\mathbf{T}_{2}\right)\end{array}$ & $\begin{array}{c}40 \mathrm{~Gy} \\
\left(\mathbf{T}_{3}\right)\end{array}$ & $\begin{array}{c}55 \text { Gy } \\
\left(\mathbf{T}_{4}\right)\end{array}$ & $\begin{array}{c}70 \text { Gy } \\
\left(\mathbf{T}_{5}\right)\end{array}$ & Mean \\
\hline \multirow[t]{2}{*}{ Yellow Golden $\left(\mathbf{V}_{1}\right)$} & 118.43 & 121.83 & 126.83 & 133.33 & 138.00 & 127.69 & 116.27 & 112.60 & 120.13 & 125.02 & 127.23 & 120.25 \\
\hline & & $(2.87)$ & $(7.09)$ & (12.58) & $(16.52)$ & & & $(-3.16)$ & $(3.32)$ & $(7.53)$ & $(9.43)$ & \\
\hline \multirow[t]{2}{*}{ Nathan $\operatorname{Red}\left(\mathbf{V}_{2}\right)$} & 104.17 & 109.00 & 118.90 & 123.63 & 126.47 & 116.43 & 104.47 & 100.37 & 108.77 & 112.35 & 116.58 & 108.51 \\
\hline & & $(4.64)$ & $(14.14)$ & (18.68) & $(21.41)$ & & & $(-3.92)$ & $(4.12)$ & $(7.54)$ & $(11.59)$ & \\
\hline \multirow{2}{*}{$\begin{array}{l}\text { White Friendship } \\
\left(\mathbf{V}_{3}\right)\end{array}$} & 109.57 & 111.43 & 120.67 & 126.57 & 132.23 & 120.03 & 105.57 & 102.32 & 112.07 & 119.73 & 121.80 & 112.30 \\
\hline & & $(1.70)$ & $(10.13)$ & $(15.52)$ & $(20.68)$ & & & $(-3.08)$ & $(6.16)$ & $(13.41)$ & $(15.37)$ & \\
\hline \multirow[t]{2}{*}{ Jester Gold $\left(\mathbf{V}_{4}\right)$} & 113.50 & 115.00 & 122.67 & 125.00 & 127.67 & 120.09 & 115.00 & 115.20 & 121.71 & 123.83 & 126.27 & 120.40 \\
\hline & & $(1.32)$ & $(8.08)$ & $(10.13)$ & $(12.48)$ & & & $(0.17)$ & $(5.83)$ & $(7.68)$ & $(9.80)$ & \\
\hline \multirow[t]{2}{*}{ American Beauty $\left(\mathbf{V}_{5}\right)$} & 113.83 & 112.83 & 122.33 & 126.80 & 134.27 & 122.01 & 101.33 & 101.83 & 110.77 & 115.77 & 118.17 & 109.57 \\
\hline & & $(-0.88)$ & $(7.47)$ & (11.39) & $(17.96)$ & & & $(0.49)$ & $(9.32)$ & $(14.25)$ & $(16.62)$ & \\
\hline \multirow[t]{2}{*}{ Red Majesty $\left(V_{6}\right)$} & 125.17 & 123.00 & 133.00 & 138.80 & 142.47 & 132.49 & 114.25 & 114.60 & 123.20 & 126.57 & 126.93 & 121.11 \\
\hline & & $(-1.73)$ & $(6.26)$ & $(10.89)$ & $(13.82)$ & & & $(0.31)$ & $(7.83)$ & $(10.78)$ & $(11.10)$ & \\
\hline \multirow[t]{2}{*}{ Purple Flora $\left(\mathbf{V}_{7}\right)$} & 103.00 & 103.50 & 111.00 & 120.17 & 123.42 & 112.22 & 95.00 & 93.17 & 100.50 & 104.83 & 109.67 & 100.63 \\
\hline & & $(0.49)$ & $(7.77)$ & (16.67) & $(19.83)$ & & & $(-1.93)$ & $(5.79)$ & $(10.35)$ & $(15.44)$ & \\
\hline \multirow[t]{2}{*}{$\operatorname{Algarve}\left(\mathbf{V}_{8}\right)$} & 113.50 & 117.33 & 124.90 & 127.73 & 130.13 & 122.72 & 109.83 & 106.05 & 114.27 & 118.90 & 123.87 & 114.58 \\
\hline & & $(3.37)$ & $(10.04)$ & (12.54) & $(14.65)$ & & & $(-3.44)$ & $(4.04)$ & $(8.26)$ & $(12.78)$ & \\
\hline Mean & 112.65 & 114.24 & 122.54 & 127.75 & 131.83 & & 107.72 & 105.77 & 113.93 & 118.38 & 121.32 & \\
\hline & \multicolumn{2}{|c|}{ CD at $5 \%$} & \multicolumn{2}{|c|}{ SEm \pm} & & & \multicolumn{2}{|c|}{ CD at $5 \%$} & \multicolumn{2}{|c|}{ SEm \pm} \\
\hline Gamma Radiation & & & \multicolumn{2}{|c|}{1.15} & \multicolumn{2}{|c|}{0.41} & & & \multicolumn{2}{|c|}{1.10} & \multicolumn{2}{|c|}{0.39} \\
\hline \multicolumn{3}{|l|}{ Varieties } & \multicolumn{2}{|c|}{1.45} & \multicolumn{2}{|c|}{0.51} & & & \multicolumn{2}{|c|}{1.40} & \multicolumn{2}{|c|}{0.50} \\
\hline \multicolumn{3}{|c|}{ Gamma Radiation * Varieties } & \multicolumn{2}{|c|}{3.24} & \multicolumn{2}{|c|}{1.15} & & & \multicolumn{2}{|c|}{3.12} & \multicolumn{2}{|c|}{1.11} \\
\hline
\end{tabular}

*Values in parentheses represent percent deviation from the control, where ( ) represent percent increase and (-) represent percent decrease 
Table.8 Effect of gamma irradiation on blooming period in different gladiolus varieties

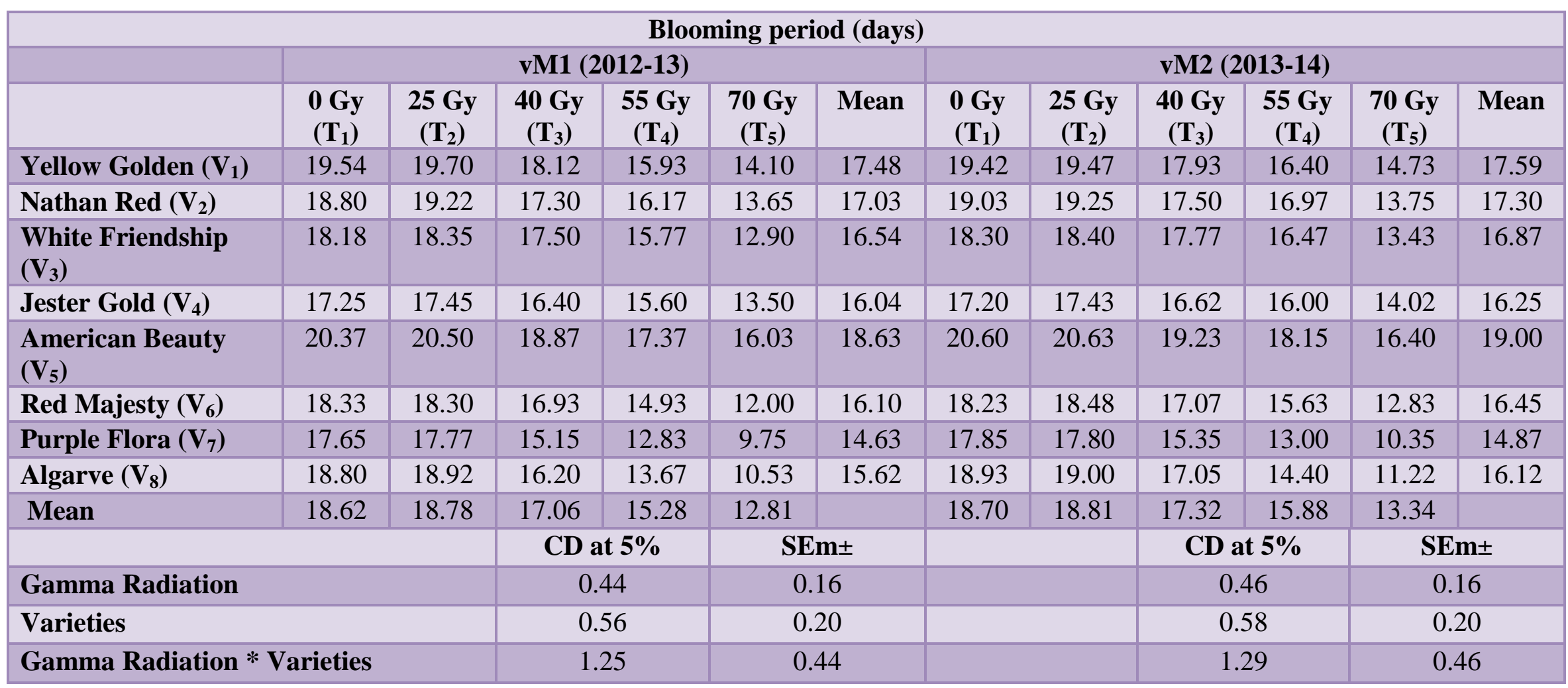


Interaction of variety White Friendship with 0 Gy exhibited maximum diameter of basal floret (11.00 and $10.83 \mathrm{~cm}$, in vM1 and vM2, respectively), whereas minimum floret diameter was recorded in interaction of variety Purple Flora with 70 Gy (6.73 and $6.78 \mathrm{~cm}$ in $\mathrm{vM} 1$ and $\mathrm{vM} 2$, respectively). This reduction may be due to the reason that gamma rays produce free radicals in cells and these radicals can damage or modify important components of plant cells and have been reported to affect differentially the morphology, anatomy, biochemistry and physiology of plants depending upon radiation level (Wi et al., 2007). The present results are in line with results of Diwedi and Banerji (2008) in Dahlia, Singh et al., (2009) in African marigold and Kumari et al., (2013) in Chrysanthemum, who recorded reduction in flower size and flower number as a result of gamma irradiation.

Non-irradiated plants resulted in longest spikes in vM1 $(99.17 \mathrm{~cm})$ generation whereas, shortest spike length was recorded in plants treated with 70 Gy gamma rays in vM1 $(51.84 \mathrm{~cm})$. In vM2 generation, maximum spike length $(101.25 \mathrm{~cm})$ was recorded in plants treated with 25 Gy gamma rays followed by control $(98.25 \mathrm{~cm})$ whereas plant treated with 70 Gy exhibited minimum spike length $(55.67 \mathrm{~cm})$. Varietal differences for spike length were also highly significant. Irrespective of gamma irradiation, spikes of variety Yellow Golden were longest i.e. 88.07 $\mathrm{cm}$ in $\mathrm{vM} 1$ as well as in $\mathrm{vM} 2$ i.e. $90.55 \mathrm{~cm}$, whereas variety Purple Flora produced shortest spikes in both the generations i.e. $68.59 \mathrm{~cm}$ in $\mathrm{vM} 1$ and $72.13 \mathrm{~cm}$ in $\mathrm{vM} 2$ generation. In vM1 generation, interaction of variety Yellow Golden with 0 Gy dose, exhibited maximum spike length $(110.63 \mathrm{~cm})$ and rachis length $(64.40 \mathrm{~cm})$, whereas interaction of variety Purple Flora with 70 Gy gamma rays dose resulted in minimum spike length $(40.83 \mathrm{~cm})$ and rachis length (19.47 $\mathrm{cm})$. Irrespective of varieties, maximum spike length $(101.25 \mathrm{~cm})$ and rachis length (59.10 $\mathrm{cm})$ was recorded at 25 Gy dose in vM2, whereas minimum at 70 Gy $(56.67 \mathrm{~cm}, 27.03$ $\mathrm{cm})$. These results are in close conformity with the findings of Patil and Dhaduk (2009) and Negi et al., (1983) who recorded that spikes were in general short with lesser number of florets in the gamma rays treated gladiolus plants. The average reduction in the parameters was also higher in the vM1 as compare to $\mathrm{vM} 2$ and these results are in conformity with the findings of Rather et al., (2002), who reported more reduction in spike length in vM1 (31.35 per cent) as compared to vM2 (15.24 per cent) generation.

The blooming period of gladiolus was decreased and there was disharmony in sequence of floret opening at higher doses of 50 and $70 \mathrm{~Gy}$. In both the generations, maximum blooming period was recorded in the plants treated with 25 Gy gamma rays (18.78 and 18.81 days, respectively in vM1 and vM2) which was at par with untreated plants (18.62 and 18.70 in vM1 and vM2) and significantly different than other treatments while plants treated with 70 Gy dose resulted in minimum blooming period (12.81 and 13.34 days in vM1 and vM2 respectively). Among the interactions, maximum blooming period was recorded in interaction of variety American Beauty with 25 Gy gamma rays dose which was at par with untreated plants of American Beauty, whereas minimum in interaction of variety Purple Flora with 70 Gy gamma rays dose which was at par with Algarve at $70 \mathrm{~Gy}$. The delay in spike emergence ultimately resulted in late blooming, which may be due to reduction in the rate of various physiological processes and inhibition of plant growth. These results are in conformity with work of Patil and Dhaduk (2009), Banerji et al., (1994) and Kumari et al., (2014) in chrysanthemum, who also reported delay in blooming after irradiation with gamma rays. 
From the present study it is concluded that gamma rays treatment can influence different floral characters in gladiolus and low doses upto $25 \mathrm{~Gy}$ can stimulate some characters whereas higher doses of 55 and 70 Gy are harmful for plant growth and floral characters. Different varieties show a difference in all qualitative and quantitative parameters under same exposure due to variation in radio sensitivity of different genotypes. Changes were less in vM2 at lower doses as compare to vM1 and persist even in vM2 at higher doses, which might be due to the diminishing effect of lower doses of gamma rays in vM2 generation and more physiological disturbances in vM1 due to immediate effect of gamma irradiation.

\section{Acknowledgement}

We acknowledge Department of Science and Technology (DST), under the Ministry of Science and Technology, Government of India for providing fellowship under the "INSPIRE programme" to carry out this research work.

\section{References}

Ashraf, M., Cheema, A.A., Rashid, M. and Qamar, Z. 2003. Effect of gamma rays on $\mathrm{M} 1$ generation in Basmati rice. Pak. J. Bot. 35: 791-795.

Bagnall, D.J., King, R.W., Whitelam, G.C., Boylan, M.T., Wagner, D. and Quail, P.H.1995. Flowering responses to altered expression of phytochrome in mutants and transgenic genes of Arabidopsis thaliana (L.) Heynh. Plant Physiol. 108(4): 495-503.

Banerji, B.K., Datta, S.K. and Sharma, S.C. 1994. Gamma irradiation studies on gladiolus cv. White Friendship. $J$. Nuclear Agric. Biol. 23(3): 127-133

Banerji, B.K., Gupta, M.N. and Datta, S.K. 1981. Effect of gamma irradiation on
Gladiolus L.-II Cytomorphological studies on Gladiolus psittacinus var Hookeri cv. Orange. NAGC Winter bulletin. pp. 50-57.

Dwivedi, A.K. and Banerji, B.K. 2008. Effect of gamma irradiation on Dahlia cv. 'Pinki' with particular reference to induction of somatic mutation. J. Orn. Hort. 11 (2): 148-151.

Jain, S.M. 2005. Major mutation-assisted plant breeding programs supported by FAO/IAEA. Plant Cell, Tissue and Organ Culture. 82: 113-123.

Kumari, K., Dhatt, K.K. and Kapoor, M. 2013. Induced mutagenesis in Chrysanthemum morifolium variety 'Otome Pink' through gamma irradiation. The Bioscan. 8(4): 14891492.

Kumari, K., Dhatt, K.K. and Singh, P. 2014. Flower colour and flower form mutants induced in Chrysanthemum morifolium through gamma irradiation. Environ. Eco. 32 (4B): 1744-1747.

Matsubara, H. 1975. Morphological investigations on tulip irradiated with gamma rays. J. Jap. Soc. Horti. Sci. 43(4): 430-442.

Patil, S.D. and Dhaduk, B.K. 2009. Effect of gamma radiation on vegetative and floral characters of commercial varieties of gladiolus (Gladiolus hybrid L.) J. Orn. Hort. 12 (4): 232-238.

Rather, Z.A., Jhon, A.Q. and Zargar, G.H. 2002. Effect of Co-60 Gamma rays on Dutch Iris-II. J. Orn. Hort. New Series. 5(2): 1-4.

Singh, V.N., Banerji, B.K., Dwivedi, A.K. and Verma, A.K. 2009. Effect of gamma irradiation on African Marigold (Tagetes erecta L.) cv. Pusa Narangi Gainda. J. Hort. Sci. 4: 36-40

Srivastava, P. and Singh, R.P. 2002. Effect of gamma radiation $(60 \mathrm{Co})$ on gladiolus. In: National Symposium on Indian Horticulture in the New Millennium. 
Lal Bagh, Bangalore 25-27.Feb 2002. Floriculture research Trends in India. R. L. Misra and S. Misra (eds.), IARI, New Delhi. Pp 239-240.

Wi, S.G., Chung, B.Y., Kim, J.S., Kim, J.H.,
Baek, M.H., Lee, J.W. and Kim, Y. S. 2007. Effect of gamma irradiation on morphological changes and biological responses in plants. Micron. 38: 553564.

\section{How to cite this article:}

Kiran Kumari, Santosh Kumar and Pragnyashree Mishra. 2019. Floral Characters of Gladiolus as Influenced by Gamma Irradiation. Int.J.Curr.Microbiol.App.Sci. 8(01): 1077-1089. doi: https://doi.org/10.20546/ijcmas.2019.801.117 